\title{
Superintendencia del Medio Ambiente, Infractor y Denunciante en el Procedimiento de Programas de Cumplimiento Ambiental
}

\author{
Environmental Agency, Offender and Complianant in the Environmental Compliance \\ Programs Procedure
}

\author{
María Victoria Galleguillos Alvear* \\ Egresada, Facultad de Derecho \\ Universidad de Chile \\ mavictoria31@gmail.com
}

\begin{abstract}
Resumen: A tres años de la puesta en marcha de la Superintendencia del Medio Ambiente, y con ello de los denominados incentivos al cumplimiento ambiental, el programa de cumplimiento se ha erguido como el instrumento más utilizado por los infractores. La alta utilización de este mecanismo, basado en la cooperación de regulado con regulador, se fundamenta principalmente en el importante beneficio que reporta para los infractores, como también en una práctica administrativa favorable a su aprobación. En este escenario, de un análisis del rol de los sujetos participantes de los procedimientos de programas de cumplimiento se concluye, que una labor activa de la SMA en la asistencia al cumplimiento, unido a una positiva ponderación de los beneficios de la herramienta por parte de los infractores, ha afianzado a los programas de cumplimiento como una opción atractiva de terminación de procedimientos sancionatorios en un sistema de fiscalización y sanción especialmente congestionado. Sin embargo, el supuesto de colaboración exclusivo entre regulado y regulador que se desprende de la normativa de los programas, se supera en la práctica, debido a la importante participación e injerencia de los denunciantes y la comunidad afectada en estos procedimientos. Lo mencionado, amerita revisar el rol de estos sujetos en estos procedimientos y, en general, en la fiscalización ambiental.
\end{abstract}

\footnotetext{
* Agradecimiento: El presente artículo presenta parte de las conclusiones de la tesis para obtener el grado licenciado en Ciencias Jurídicas y Sociales de las Universidad de Chile titulada "Cooperación en Medio Ambiente: un análisis del desempeño de los programas de cumplimiento ambiental" guiada por el profesor Luis Cordero Vega, tesis que se enmarca dentro del Fondecyt número 1161741 "Análisis y Revisión Dogmática del Derecho Administrativo Sancionador en Chile a partir de su parte especial" del cual el profesor Luis Cordero es co-investigador.
} 
Galleguillos - Superintendencia del Medio Ambiente, Infractor y Denunciante en el Procedimiento de Programas de Cumplimiento Ambiental • Sección Doctrina

Palabras Clave: programa de cumplimiento, infractores, Superintendencia del Medio Ambiente, denunciante.

Abstract: Three years from the implementation of the Environmental Agency along with the so-called incentives for environmental compliance, the Compliance Program has become the most commonly used device by the offenders. The high utilization of this mechanism, which is based upon regulatory cooperation with the regulator, is explained both by the important benefit that it signifies to the offenders, and the common administrative practice to favor its approval. In this scenario, an analysis of the role of the subjects taking part in compliance program procedures concludes that an active work of the Environmental Agency in compliance assistance along with a positive weighting of the benefits of the tool by offenders, has strengthened Compliance Programs as an attractive option to terminate sanctioning procedures in a particulary congested enforcement and sanctioning system. However, the scenario of exclusive collaboration between the regulated and the regulator that is set forth in the programs regulation, is exceeded in practice by the significant participation and interference of the complainants and the community affected in these procedures, which shows the importance of revisiting the role of these actors in these procedures and in environmental audit in general.

Key words: Compliance Programs, Offenders, Environmental Agency, Complianant.

\section{Introducción}

La regulación orgánica de la Superintendencia de Medio Ambiente (en adelante "SMA"), a diferencia de la mayoría de las regulaciones de órganos administrativos con potestades fiscalizadoras y sancionadoras, contempla un párrafo completo destinado exclusivamente al procedimiento administrativo sancionador (artículos 47 a 54 de la Ley Orgánica de la Superintendencia del Medio Ambiente, en adelante "LO-SMA) ${ }^{1}$. Dentro de las explicaciones que podemos encontrar a este procedimiento especialmente reglado, se encuentra que la SMA en tanto órgano que concentra las funciones de fiscalización y sanción ambiental, fue considerada el corazón de la reforma introducida por la Ley $\mathrm{N}^{\circ}$ 20.417 en el año 2010, modificación normativa e institucional motivada principalmente por la existencia de un modelo de fiscalización altamente ineficiente ${ }^{2}$. Es también integrante de esta explicación el hecho de que el derecho administrativo sancionador (en adelante "DAS") en materia ambiental constituye un imperativo para el cumplimiento del deber estatal establecido en el artículo 19 n 8 de la Constitución, en especial atención a la

\footnotetext{
${ }^{1}$ Jorge, Bermúdez, "Fundamento y límites de la potestad sancionadora administrativa en materia ambiental", Revista de Derecho de la Pontificia Universidad Católica de Valparaíso, №.40 (2013): 450.

2 Biblioteca del Congreso Nacional. Historia de la Ley $\mathrm{N}^{\circ} 20.417$ (2010): 13, http://www.bcn.cl/obtienearchivo?id=recursoslegales/10221.3/3929/6/HL20417.pdf.
} 
habilitación expresa que contempla la Carta Fundamental de establecer restricciones específicas al ejercicio de determinados derechos, en el inciso segundo del mencionado artículo $^{3}$. Por último, la inclusión de mecanismos novedosos en la regulación nacional del DAS, como son los incentivos al cumplimiento ambiental (en adelante "ICA"), explican la robustez de la regulación de este procedimiento.

Los ICA -programas de cumplimiento, autodenuncia y planes de reparación ${ }^{4}$ incorporados en la LO-SMA, se enmarcan dentro de un fenómeno relativamente reciente en la normativa nacional que apunta a la incorporación de mecanismos de cooperación entre el Estado y privados con el fin de generar condiciones óptimas para el cumplimiento de la legislación. Lo adopción de estos mecanismos se encuentra, en gran parte, justificado por la imposibilidad del Estado de contar con suficientes inspectores, recursos estatales de fiscalización y posibilidades de apertura de procedimientos sancionatorios ${ }^{5}$, como también por la expansión de la actividad económica regulada y con ello la creciente complejización de las regulaciones administrativas ${ }^{6}$. En definitiva, la incorporación de estos mecanismos se encuentra especialmente justificada en la consecución de una mayor eficiencia y eficacia en la gestión pública ${ }^{7}$. En este sentido, se ha declarado que los ICA tienen por objetivo "incentivar la participación de la ciudadanía en pos del cumplimiento de la normativa ambiental" $"$. Asimismo, y acentuando la perspectiva colaborativa de estos instrumentos, se ha indicado que éstos responden a la necesidad de "crear mecanismos legales centrados en la prevención y promoción del cumplimiento, más que en el efecto disuasivo de la fiscalización y sanción de infracciones medioambientales"?.

En concreto, el programa de cumplimiento ambiental (en adelante "PdC") es un instrumento que otorga al infractor, de la normativa ambiental o de una autorización administrativa, la posibilidad de presentar un plan de acciones destinado a corregir su actuar ilegal -en un plazo definido y bajo la vigilancia de la autoridad- y en caso de cumplirlo satisfactoriamente, eximirse totalmente de sanción. En suma, es en la institución de los PdC donde subyace una verdadera concepción normativa, en la cual prevalece el valor de un pronto y cautelado regreso al respeto de la norma por sobre la imposición de la sanción. Con todo, la relevancia de los PdC no solo se circunscribe a aspectos teóricos dentro del actual DAS ambiental. Este instrumento, también se ha erguido como el ICA

\footnotetext{
${ }^{3}$ Jorge Bermúdez, Fundamentos de Derecho Ambiental (Valparaíso: UV Ed., Segunda Edición, 2014 ), 450.

${ }^{4}$ También es posible considerar como incentivos al cumplimiento ambiental el Registro Público de Sanciones y la función de asistencia al cumplimiento de la SMA.

5 Iván Poklepovic, “Análisis Crítico del Sistema de Incentivos al Cumplimiento Ambiental de la Ley 20.417, que crea la Superintendencia del Medio Ambiente," en Actas de las V Jornadas de Derecho Ambiental, Derecho Ambiental en tiempos de reformas (2010), 176.

6 Brigitte Leal, "Planificación, colaboración y autocontrol: nuevos instrumentos normativos para la administración fiscalizadora,” Revista de Derecho Público, Vol. 83 (Segundo Semestre, 2015): 106.

${ }^{7}$ Ibíd. 107.

${ }^{8}$ Jean Paul Dussaubat, "Daño ambiental y el fracaso de la reparación voluntaria: diagnóstico y propuestas," Revista de Derecho Ambiental, Centro de Derecho Ambiental, №6 (2016): 184.

${ }^{9}$ Jorge Ossandón, Incentivos al Cumplimiento Ambiental (Santiago: LibroMar, 2015), 202.
} 
Galleguillos - Superintendencia del Medio Ambiente, Infractor y Denunciante en el Procedimiento de Programas de Cumplimiento Ambiental • Sección Doctrina

más utilizado por los agentes regulados, presentándose a la fecha de esta investigación 181 $\mathrm{PdC}^{10}$.

En este contexto, el objetivo de este trabajo, será analizar el rol que han tenido hasta el momento la SMA, los infractores y los denunciantes en los procedimientos de programas de cumplimiento (en adelante "PPdC"). Lo anterior, con el propósito de determinar cuáles han sido los factores que explican la alta aplicación de este mecanismo, como también si el rol que la normativa les otorga a los distintos actores se condice con la praxis que ha tenido este instrumento en los procedimientos administrativos sancionatorios llevados por la SMA.

\section{Algunas consideraciones generales sobre el PdC Ambiental}

El PdC ambiental constituye uno de los tres incentivos al cumplimiento -los dos restantes corresponden a los planes de reparación y la autodenuncia- introducidos por la LO-SMA. El artículo 42 de la antedicha ley, y el Decreto Supremo $N^{\circ}$ 30/2012 del Ministerio del Medio Ambiente, que aprueba reglamento sobre programas de cumplimiento, autodenuncia y planes de reparación (en adelante "DS.30"), regulan la oportunidad de presentación, definición, contenidos mínimos, requisitos de aprobación e impedimentos para su utilización.

El PdC se alza como una de las alternativas -siendo la otra la presentación de descargos- que la ley le otorga al presunto infractor en un procedimiento administrativo sancionador frente a la SMA cuando ésta realiza una formulación de cargos (en adelante "FC"). El artículo 42 contempla una definición legal de programas de cumplimiento señalando que: "se entenderá como programa de cumplimiento, el plan de acciones y metas presentado por el infractor, para que, dentro de un plazo fijado por la Superintendencia, los responsables cumplan satisfactoriamente con la normativa ambiental".

El principal objetivo del PdC es el cumplimiento de la normativa ambiental, junto con la reducción o eliminación de los efectos negativos derivados del incumplimiento. Sin embargo, esta herramienta tiene a su vez objetivos secundarios referidos a una utilización más eficiente de los recursos de fiscalización, generación de espacios de cooperación entre el regulador y los regulados, como también instalación de prácticas de cumplimiento normativo.

De la regulación del PdC es posible concluir que estamos frente a un instrumento con las siguientes características: i) reactivo, ii) establecido en beneficio del infractor, (iii) de alcance restringido ${ }^{11}$, iv) temporal, v) necesariamente refrendado por la autoridad para

\footnotetext{
${ }^{10}$ El número total de programas de cumplimiento es 181 a la fecha de finalización de esta investigación (9 de diciembre de 2016), SNIFA.

${ }^{11} \mathrm{El}$ alcance restringido del PdC se refiere a que este nace a propósito de la formulación de cargo realizada por la SMA y muere con el restablecimiento de la legalidad del actuar ilegal en ellos establecido. Así, de acuerdo a la Guía para la presentación de Programas de Cumplimiento de la SMA no son acciones propias de un PdC aquellas "acciones que no se relacionen con los hechos contenidos en la resolución de formulación de
} 
cumplir sus efectos e vi) intraproceso. El carácter reactivo, como también el que la herramienta sea una opción a favor del infractor, son los elementos que diferencian más intensamente al PdC ambiental de otros programas de cumplimiento regulados por la normativa nacional, especialmente los programas penales ${ }^{12}$ y de libre competencia ${ }^{13}$, los cuales corresponden a instituciones jurídicas orientadas eminentemente a la prevención de ilícitos ${ }^{14}$.

La ley orgánica de la SMA, en su artículo 42, entregó a la potestad reglamentaria la regulación de los criterios de aprobación de los $\mathrm{PdC}$, remisión que fue materializada en el DS.30. El artículo 9 del citado reglamento establece los criterios de aprobación del PdC, los cuales pueden clasificarse en criterios positivos y negativos. Los criterios positivos de aprobación corresponden a aquellas características que los $\mathrm{PdC}$ deben cumplir necesariamente para efectos de satisfacer los fines propuestos por la normativa para este instrumento, éstos son: integridad, eficacia y verificabilidad. Por otro lado, los criterios negativos de aprobación no corresponden a características del $\mathrm{PdC}$, sino que guardan relación con un control de un uso conforme a derecho del instrumento, y por ello están destinados a evitar una utilización abusiva, fraudulenta o contraria a ley del mismo. Estos elementos dicen relación con la utilización del $\mathrm{PdC}$ para eludir responsabilidad, aprovecharse de la infracción o utilizarlo con fines manifiestamente dilatorios. La principal diferencia entre criterios positivos y negativos radica en que los primeros corresponden a elementos esencialmente perfectibles, mientras que los segundos son, en todo evento, impeditivos a la aprobación del PdC.

En relación a la naturaleza jurídica del programa de cumplimiento, Guzmán Rosen los califica como una forma extraordinaria de poner término al procedimiento sancionatorio $^{15}$. Al respecto, el Segundo Tribunal Ambiental, en un sentido similar, ha señalado que "procedimentalmente, el programa de cumplimiento es una forma anormal de terminar el procedimiento administrativo sancionador" ". La calificación del PdC como una forma extraordinaria o anormal de poner término al procedimiento sancionatorio es pertinente en términos procedimentales, sin embargo, esta calificación solo es acertada en los casos de ejecución satisfactoria del PdC, ya que, en casos de incumplimiento, el PdC solo constituye un motivo para la suspensión del procedimiento sancionatorio.

cargos o sus efectos", estas medidas son en efecto catalogadas como acciones inconducentes o manifiestamente dilatorias, por la misma guía.

${ }^{12}$ La Ley $N^{\circ} 20.393$ sobre Responsabilidad Penal de las Personas Jurídicas por delitos de cohecho de funcionario público nacional o extranjero, lavado de activos y financiamiento del terrorismo, regula en su artículo $4^{\circ}$ los modelos de prevención de delitos, denominados también programas de cumplimiento penales.

${ }^{13}$ Fiscalía Nacional Económica, Material de Promoción Nº3 referido a Programas de Cumplimiento en materia de Libre Competencia (Santiago: 2012).

${ }^{14}$ En relación a este punto, Gonzalo Medina ha manifestado que el PdC ambiental sería un mecanismo más centrado en la mitigación que en la prevención. Gonzalo Medina, Efectos del Compliance, Incentivos para un Buen Ciudadano Corporativo, La Semana Jurídica, Thomson Reuters, Año II, Número 63, 2013.

15 Rodrigo Guzmán, Derecho Ambiental Chileno. Principios, instituciones e instrumentos de gestión (Santiago: Planeta Sostenible, 2012), 213.

${ }^{16}$ Segundo Tribunal Ambiental, Rol No 68-2015, Considerando Vigésimo Segundo. 
Galleguillos - Superintendencia del Medio Ambiente, Infractor y Denunciante en el Procedimiento de Programas de Cumplimiento Ambiental • Sección Doctrina

En cuanto a la naturaleza no procedimental del programa de cumplimiento, estimamos que se trata de un instrumento de gestión ambiental ${ }^{17}$, entendiendo por tal el "conjunto de medidas de variado orden destinadas al logro de finalidades de protección y mejoramiento ambiental"18. Particularmente, estamos ante un instrumento de gestión que se funda en la cooperación de los sujetos regulados con la administración, y que es fiscalizable -al igual que un importante número de los instrumentos de gestión establecidos en el título II de la ley 19.300- por la SMA.

\section{El rol de la SMA en los PPdC}

La SMA es actualmente el órgano clave en la cautela del cumplimiento de la normativa ambiental. En su génesis, como ya ha sido señalado, se buscó potenciar no solo las facultades sancionatorias, sino especialmente un rol preventivo y promotor de mejores prácticas. Siguiendo este ánimo, la SMA ha sido caracterizada como una "entidad no necesariamente 'persecutora' sino más bien que incentiva el cumplimiento con potentes instrumentos"19. En efecto, el artículo $3^{\circ}$, que enumera las funciones y atribuciones del organismo, complejiza las funciones de la institución en atención a su labor de asistencia a los regulados, obligando, por ejemplo en la letra (s), a que las normas e instrucciones dictadas estén sistematizadas de tal forma de facilitar su acceso y conocimiento. En esta misma línea, el antedicho artículo contempla una función destinada exclusivamente a esta labor. Así, la letra (u) señala que la SMA deberá “(p)roporcionar asistencia a sus regulados para la presentación de planes de cumplimiento o de reparación, así como para orientarlos en la comprensión de las obligaciones que emanan de los instrumentos individualizados en el artículo $2^{\circ}$ de esta ley".

En el particular caso de los PdC, la SMA ha concretado su función de proporción de asistencia principalmente a través de dos mecanismos: la dictación de la i) guía para la presentación de programas de cumplimiento ambiental por infracciones a instrumentos de carácter ambiental ${ }^{20}$ (en adelante "GpPdC"), y la implementación de ii) reuniones de asistencia en el PPdC.

La GpPdC constituye un "material de apoyo y guía en el proceso de presentación de un Programa de Cumplimiento" 21 . El documento, junto con concentrar la normativa

\footnotetext{
${ }^{17}$ La SMA en sus resoluciones ha manifestado esta postura al mencionar que "los programas de cumplimiento son un instrumento de gestión ambiental que permite al titular volver cumplimiento en un plazo acotado, mediante la realización de acciones concretas e íntegras que se hagan cargo de manera eficaz de todas y cada uno de los hechos infraccionales detectados y que, a su vez puedan ser verificables en el tiempo" F-0382013/Municipalidad de Temuco.

${ }^{18}$ Jorge Bermúdez, Fundamentos de Derecho Ambiental, Op Cit., 192.

19 Marie Claude Plumer, "Los Tribunales Ambientales: se completa la reforma a la institucionalidad ambiental," Anuario de Derecho Público, Universidad Diego Portales (2013): 310.

${ }^{20}$ Superintendencia del Medio Ambiente, "Guía para la Presentación de Programas de Cumplimiento Ambiental por Infracciones a Instrumentos de Carácter Ambiental,” julio 2016, http://www.sma.gob.cl/index.php/documentos/documentos-de-interes/documentos/guias-sma.

${ }^{21} \mathrm{Ib} ., 3$.
} 
aplicable de los PdC y formularios destinados a facilitar ciertas solicitudes durante el PPdC, aclara algunos aspectos no regulados por la normativa y solucionados en la praxis administrativa. En este sentido, son particularmente relevantes las aclaraciones relativas a la incompatibilidad del PdC en caso de infracciones que hayan causado daño ambiental ${ }^{22}$, la imposibilidad de presentar acciones indemnizatorias o resarcitorias como integrantes de un $\mathrm{PdC}^{23}$, como también los límites del PdC para modificar de forma permanente una $\mathrm{RCA}^{24}$.

En relación a la reunión de asistencia, ésta se irgue como un mecanismo altamente utilizado en la práctica. La GpPdC indica que las reuniones de asistencia tienen por objetivo "proporcionar asistencia a los sujetos regulados sobre los requisitos y criterios para la presentación y aprobación de PdC así como en la comprensión de las obligaciones que emanan de este instrumento" ${ }^{25}$. A pesar de la regulación existente de reuniones de asistencia, igualmente consta en expedientes administrativos que existen mecanismos no formalizados de asistencia por medio de comunicaciones con el fiscal instructor ${ }^{26}$.

La reunión de asistencia constituye un importante instrumento para concretar la cooperación que subyace a esta herramienta. Sin embargo, para que ello sea posible, es imprescindible que los participantes de la reunión tengan las potestades necesarias para tomar decisiones en relación al PdC, como también manejen información suficiente para emitir pronunciamientos fundados acerca de los aspectos técnicos y jurídicos que pueden ser objeto de estas reuniones. Cumpliendo estas características, estas reuniones podrán ser una instancia de verdadera colaboración, y no simplemente un espacio de aclaración vertical de los aspectos necesarios para la aprobación del programa por la autoridad.

La SMA no solo tiene un rol esencial en la implementación de los $\mathrm{PdC}$ en lo que se refiere a su labor de asistencia, sino que también resulta particularmente relevante su actuación en las etapas de aprobación y fiscalización del instrumento.

En la fase de aprobación la SMA, por medio de su División de Sanción y Cumplimiento, ha adoptado la práctica de establecer observaciones y correcciones de oficio a los PdC presentados por los sujetos formulados de cargos. Dentro de las razones que son otorgadas por la SMA para realizar observaciones a los PdC, se encuentra la exigencia de que el programa de cumplimiento "cumpla cabalmente los criterios establecidos reglamentariamente para su aprobación, a saber integridad, eficacia y verificabilidad" 27 , la detección de "ciertas deficiencias en la determinación de metas, objetivos, plazos y medios de verificación que no lograrían satisfacer los requisitos de forma propios de todo programa" 28 , como también la necesidad de subsanar ciertos aspectos "a fin de garantizar el

\footnotetext{
${ }^{22} \mathrm{Ib} ., 6$.

${ }^{23} \mathrm{Ib} ., 13$.

${ }^{24} \mathrm{Ib} ., 14$.

${ }^{25} \mathrm{Ib}, 7$.

${ }^{26}$ Véase para tal efecto, Superintendencia del Medio Ambiente, Res. Ex. Nº/ D-038-2015/Planta Costa Sur S.A.

27 Véase para tal efecto, Superintendencia del Medio Ambiente, Res. Ex No4 /D-012-2016/Agrícola Chorombo S.A.

${ }^{28}$ Véase para tal efecto, Superintendencia del Medio Ambiente, Res. Ex N³/ D-002-2015/Ecomaule S.A.
} 
Galleguillos - Superintendencia del Medio Ambiente, Infractor y Denunciante en el Procedimiento de Programas de Cumplimiento Ambiental • Sección Doctrina

correcto seguimiento y fiscalización" 29 . El argumento expuesto de forma reiterada por la SMA, para no rechazar el programa a pesar de sus deficiencias, es que el instrumento presentado captaría "la esencia de esta institución en el sentido de que se hace cargo del hecho constitutivo de infracción" "30 y por ello se otorga un plazo, de 5 a 8 días hábiles desde la notificación del acto administrativo que formula observaciones, para presentar un nuevo programa de cumplimiento que las incluya debidamente, so pena de que el programa sea rechazado, reiniciando procedimiento sancionatorio. Se registran casos en los cuales el PdC es rechazado por no acoger las observaciones apropiadamente ${ }^{31}$, como también casos en los cuales la SMA observa un PdC que ya fue objeto de observaciones anteriormente ${ }^{32}$.

Las correcciones de oficio se diferencian de las observaciones, dado que estas no suponen que el sujeto formulado de cargos despliegue actividad alguna, y tampoco condicionan la entrada en vigencia del PdC. En cuanto al contenido de las correcciones de oficio, éstas son de menor complejidad que las observaciones, y en ningún caso se refieren a aspectos no considerados en el PdC presentado.

La realización de observaciones y correcciones de oficio por la SMA es una práctica que debiese ser realizada con especial cautela, dado que pueden tener por resultado un PdC con acciones a las cuales el titular no puede obligarse o que no se condicen con la realidad del proyecto o actividad. Al respecto, cabe recordar que la institución del programa se sustenta en un reconocimiento al conocimiento privilegiado que tiene el formulado de cargos de su actividad, lo que le permite proponer la mejor forma de volver a un estado de cumplimiento. En este escenario, el control de la SMA debiese concentrarse especialmente en los criterios de aprobación de verificabilidad e integridad, existiendo mayor deferencia en lo que respecta a la eficacia de las medidas presentadas.

La SMA es el organismo encargado de fiscalizar la ejecución de los PdC por medio de su División de Fiscalización, de acuerdo a lo establece el artículo 3 del DS.30. La antedicha división emite el Informe de Fiscalización Ambiental del Programa de Cumplimiento (en adelante "IFA"), en el cual se realiza una revisión de la información aportada por la empresa, en el marco del cumplimiento del plan de acciones establecido en el PdC. Es a partir de este informe, que se fundamentan la mayor parte de las resoluciones que reinician el procedimiento sancionatorio, debido a un incumplimiento del plan propuesto en el infractor. Asimismo, el IFA sirve de sustento principal para una declaración de ejecución satisfactoria del instrumento, y con ello de la terminación del procedimiento sancionatorio.

A la fecha, se registran tan solo 3 casos de incumplimiento, los cuales corresponden a menos del $2 \%$ de los programas aprobados por la autoridad. Estos casos se encuentran

\footnotetext{
${ }^{29}$ Véase para tal efecto, Superintendencia del Medio Ambiente, Res. Ex. No 4 / F-003-2016/ Compañía exploradora y explotadora de minas chileno rumana.

30 Véase para tal efecto, Superintendencia del Medio Ambiente, Res. Ex.N2/ D-013-2015/Salmones Camanchaca S.A, Res. Ex.Nº4/ D-067-2015/Empresa de Servicios Sanitarios Los Lagos S.A., Res. Ex. No3/D-070-2015/Demarco S.A.

${ }^{31}$ Véase para tal efecto, Superintendencia del Medio Ambiente, F-038-2013/Municipalidad de Temuco.

${ }^{32}$ Véase para tal efecto, Superintendencia del Medio Ambiente, F-030-2015/RESAM S.A.
} 
caratulados bajo los expedientes D-002-2015/Eco Maule; D-005-2015/Hotel Magnolia Huérfanos y D-027-2014/Autodromo Codegua. En relación al estándar de incumplimiento necesario para reiniciar el procedimiento sancionatorio, se evidencia que en los tres casos la SMA justifica su decisión en un incumplimiento grave, reiterado y/o variado del instrumento. Así, pareciera que para la SMA los incumplimientos puntuales, aislados o de menor relevancia del PdC no tendrían la aptitud suficiente para provocar el reinicio del procedimiento sancionatorio.

Debido al escaso número de casos, es difícil verificar con certeza cuál es el estándar utilizado por la SMA para decretar el incumplimiento de un PdC. A nuestro juicio, si bien un incumplimiento puntual y de escasa relevancia del instrumento pareciera no ser apto para reiniciar el procedimiento sancionatorio, el estándar de diligencia exigido por la SMA -tal como el mismo organismo lo ha declarado ${ }^{33}$ - debiese ser alto, y por ello no debieran ser tolerados mayores incumplimientos del plan de acciones definido por el titular. Lo anterior es esencial para legitimar el uso de la herramienta, como también para cumplir los fines del instrumento.

\section{El rol de los infractores en los PPdC}

Los ICA son instrumentos puestos a disposición del infractor, y su utilización implica, en definitiva, un hecho voluntario. Es por ello que su rol activo es un requisito sine qua non para su éxito. La lógica de los ICA consiste en otorgar ciertos beneficios a disposición del infractor, destinados a influir en un comportamiento coincidente con la normativa y, así, obtener una mejor y más efectiva consecución de los fines perseguidos por la administración con la misma. Lo anterior, ha sido refrendado por el Segundo Tribunal Ambiental al indicar que el objetivo principal de los mecanismos de incentivo al cumplimiento es "el establecimiento de alternativas beneficiosas para ambas partes"34.

En el caso del PdC, el incentivo otorgado al infractor se traduce principalmente en la eximición de sanción en casos de ejecución satisfactoria, pero también, en la posibilidad de reconducir la actividad o proyecto al cumplimiento normativo en un plazo acotado, con la asistencia de la autoridad estatal, lo cual dota dicha reconducción de una mayor seguridad y certeza.

Sin embargo, para los infractores, los ICA funcionan bajo una lógica de zanahoria y garrote, así en el caso particular del PdC, su incumplimiento conlleva, de acuerdo al inciso final de artículo 40 de la LO-SMA, la posibilidad de aplicar "hasta el doble de la multa que corresponda a la infracción original dentro del rango señalado en la letra b) del artículo 38".

\footnotetext{
${ }^{33}$ Véase para tal efecto, Superintendencia del Medio Ambiente, considerando 56 de la Res. Ex. No5/D-0052015/Gastón de las Riberas.

${ }^{34}$ Segundo Tribunal Ambiental, Rol No 68-2015, Considerando Decimoctavo.
} 
Galleguillos - Superintendencia del Medio Ambiente, Infractor y Denunciante en el Procedimiento de Programas de Cumplimiento Ambiental • Sección Doctrina

Como ya ha sido mencionado, el PdC ha sido una alternativa altamente utilizada en la práctica, alcanzando un $40 \%{ }^{35}$ de los procedimientos sancionatorios ante la SMA. Así, en un análisis de riesgos, los sujetos regulados han ponderado, en un número importante de casos, que los beneficios otorgados por el programa son superiores a sus costos.

Pese a la alta utilización de $\operatorname{los} \mathrm{PdC}$, existen límites a su uso. Estos límites corresponden, por una parte, a circunstancias impeditivas referidas al historial del infractor, las cuales consisten: i) un acogimiento previo al mecanismo de programas de gradualidad, ii) a los sujetos ya sancionados por infracciones gravísimas como también aquellos iii) infractores que ya se hayan visto beneficiados por la institución del $\mathrm{PdC}$ en casos de infracciones graves o gravísimas. Por otro lado, también existen circunstancias fácticas del sujeto formulado de cargos que pueden dificultar o impedir la presentación de un programa. Estos elementos se refieren especialmente a los importantes costos que supone una presentación oportuna de un $\mathrm{PdC}^{36}$ con los estándares exigidos por la ley. Al respecto, cabe destacar que, si bien la SMA ha adoptado acciones destinadas a facilitar la utilización de esta herramienta, no existen medidas especialmente enfocadas en infractores de menor tamaño, salvo la Guía para la presentación de un Programa de Cumplimiento Infracciones a la norma de emisión de ruidos, Infractores de Menor Tamaño ${ }^{37}$.

El sujeto formulado de cargos es quien define principalmente el contenido del PdC, estableciendo las acciones y metas, plan de seguimiento, información técnica y costos del instrumento. La relevancia del rol del sujeto formulado de cargos es esencial para que esta herramienta sea efectivamente una concreción del principio colaborativo. Lo anterior, dado que la institución del $\mathrm{PdC}$ reconoce que es el regulado quien posee el mayor grado de conocimiento de su actividad o proyecto, lo que le permite proponer a la SMA la forma más efectiva de volver al cumplimiento de la norma.

Tal como ya fue mencionado, el porcentaje de incumplimiento del PdC es bajo. Esta circunstancia podría fundarse en las exiguas capacidades de fiscalización de la SMA. Sin embargo, dado que los PdC contemplan medios de verificación, la labor fiscalizadora del organismo en estos casos facilita de forma sustancial, y por ello, no es arriesgado establecer que es probable que estos instrumentos sean efectivamente observados por los infractores de forma satisfactoria en la mayoría de los casos.

\section{El rol del denunciante en los PPdC}

\footnotetext{
35 De un total de 446 procedimientos sancionatorios iniciados por la SMA, 148 tienen un programa de cumplimiento en ejecución, 30 ya han sido finalizados con $\mathrm{PdC}$ satisfactorio, y 3 han contemplan un programa de cumplimiento terminado insatisfactoriamente (181 en total).

36 La única oportunidad procesal para presentar un $\mathrm{PdC}$-tanto en procedimientos asociados a una autodenuncia como aquellos iniciados por la SMA- es la regulada por el artículo 42 inciso segundo, es decir en el plazo de 10 días tras la formulación de cargos. Sin embargo, cabe destacar, que se ha instalado una práctica de solicitudes de ampliación de plazo, solicitud que en prácticamente todos los casos en acogida sin mayores fundamentos.

37 Superintendencia del Medio Ambiente, "Guía para la presentación de un Programa de Cumplimiento Infracciones a la norma de emisión de ruidos, Infractores de Menor Tamaño" http://www.sma.gob.cl/index.php/documentos/documentos-de-interes/documentos/guias-sma.
} 
De acuerdo al artículo 47 de la LO-SMA, una de las formas de iniciar el procedimiento administrativo sancionatorio ambiental es por medio de una denuncia formulada en los términos y con los requisitos contemplados por la misma disposición legal. De acuerdo a la Cuenta Pública de la SMA, la institución recibe aproximadamente 1.500 denuncias anualmente ${ }^{38}$. Al respecto, el Superintendente Cristián Franz, ha declarado públicamente que la SMA "no tiene la capacidad de procesar todas las denuncias que ingresan" 39 y que por ello se ven obligadas a priorizarlas, dando urgencia a aquellas que "digan relación con afectación directa a la población o situaciones que hayan generado un daño ambiental" 40 , dejando en claro que no se cuenta con el "personal necesario para alcanzar el estándar de físcalización de un país como Chile"41. Las declaraciones del Superintendente son coincidentes con el diagnóstico que motivó la creación de los ICA como herramientas de colaboración, orientadas a una mejor utilización de los recursos públicos, en un contexto de creciente demanda por injerencia estatal, especialmente en materias de fiscalización. Sin embargo, el supuesto de colaboración previsto por la normativa está constituido exclusivamente por el infractor y la SMA, no considerando en dicho esquema al denunciante o a la comunidad afectada, como un potencial ente colaborador en los PPdC.

La relevancia de la participación de la ciudadanía en materia ambiental ha sido remarcada por la jurisprudencia ${ }^{42}$ como por la doctrina ${ }^{43}$, especialmente en atención a las reformas en participación ciudadana introducidas por la Ley $\mathrm{N}^{\circ} 20.417$. Lo anterior ha sido destacado con especial vigor en el último periodo, debido al proceso de negociación de un acuerdo regional sobre acceso a la información, participación pública y acceso a la justicia en asuntos ambientales en América Latina y el Caribe, basado en el principio 10 de la Declaración de Río, en el cual Chile ha tenido un rol protagónico ${ }^{44}$. Sin embargo, en lo que respecta a la fiscalización ambiental, el rol que recae en la ciudadanía solo ha sido desarrollado normativamente en una intensificación del acceso a la información ambiental, mediante el establecimiento Sistema Nacional de Información de Fiscalización Ambiental,

\footnotetext{
${ }^{38}$ La Cuenta Pública de la SMA del año 2015 desglosa la presentación de denuncias anualmente indicando que: 2013 se presentaron 1.484 denuncias, 2014 se presentaron 1.501 denuncias y el 2015 se presentaron 1.559 denuncias.

39 Superintendencia de Medioambiente informó sobre resultados a la fecha y operatividad del sistema, https://www.camara.cl/prensa/noticias_detalle.aspx?prmid=129753.

${ }^{40} \mathrm{Ib}$.

${ }^{41} \mathrm{Ib}$.

${ }^{42}$ Corte Suprema, Causa Rol N ${ }^{\circ}$ 1.119-2015. Fernando Dougnac y otros con Ministerio del Medio Ambiente. 30 de septiembre de 2015.

${ }^{43}$ Camilo Mirosevic, "La participación ciudadana en el procedimiento de evaluación de impacto ambiental y las reformas introducidas por la Ley N²0.417," Revista de Derecho de la Pontificia Universidad Católica de Valparaíso Vol. XXXVI, N 1 (2011): 281- 323 y Ezio Costa y Paula Fuentes, "La participación ciudadana en las declaraciones de impacto ambiental,” Revista Justicia Ambiental Vol. III (2011): 83-106.

${ }^{44}$ Ministerio del Medio Ambiente, "Acuerdo Regional Liderado por Chile espera dar mayor acceso a la ciudadanía a información y justicia ambiental” http://portal.mma.gob.cl/acuerdo-regional-liderado-por-chileespera-dar-mayor-acceso-a-la-ciudadania-a-informacion-y-justicia-ambiental/.
} 
Galleguillos - Superintendencia del Medio Ambiente, Infractor y Denunciante en el Procedimiento de Programas de Cumplimiento Ambiental • Sección Doctrina

como también en la regulación de la denuncia, mas no se le han otorgado mayor participación o herramientas en los procedimientos sancionatorios o en la físcalización ambiental de instrumentos.

Aproximadamente, un $40 \%$ de los procedimientos de $\mathrm{PdC}$ fueron iniciados por vía de una denuncia ${ }^{45}$. Sin embargo, la Ley $\mathrm{N}^{\circ} 20.417$, el D.S 30 y la GpPdC no contemplan al denunciante en el PPdC. La ausencia de una regulación especial para que denunciantes, o la comunidad afectada, pueda participar o tener injerencia en el programa, ha sido considerada como una "vulneración de las normas de participación ciudadana que se han impuesto con fuerza en la normativa ambiental chilena" 46 . En una línea similar, se han observado las complejidades que tiene este instrumento en lo que respecta a su validación por parte de la ciudadanía directamente afectada, debido a que, en casos de desempeño satisfactorio del PdC, no concurre sanción alguna por los incumplimientos ${ }^{47}$. Considerando estas observaciones, durante la tramitación legislativa se propuso incorporar la posibilidad de oposición al PdC por cualquier tercero interesado o afectado por la infracción ${ }^{48}$, lo que, sin embargo, no fue incluido en el texto final de la ley.

Ante la ausencia de una normativa especial, se ha aplicado supletoriamente la Ley $\mathrm{N}^{\circ}$ 19.880, de Bases de los procedimientos administrativos (en adelante "LBPA"), la cual, en tanto norma de garantías básicas, ha permitido que el denunciante pueda participar en estos procedimientos. Especialmente, el principio de contradictoriedad, establecido en el artículo 10 de la LBPA, es el que ha permitido esta actuación. El principio en cuestión proviene del origen garantista del procedimiento administrativo, y obliga a la administración a admitir las controversias presentadas de todas aquellas situaciones en que se encuentren vinculados los particulares ${ }^{49}$. Así, en virtud de este principio, la SMA ha debido necesariamente ponderar los elementos incorporados por los denunciantes en los procedimientos de PdC.

En la práctica, los denunciantes en tanto interesados en el procedimiento sancionatorio -calidad otorgada expresamente por el artículo 21 de la LO-SMA- han participado de diversas formas en los PPdC. Entre ellas, es posible destacar su i) injerencia en la determinación del contenido del PdC, -primordialmente a través de la realización de

\footnotetext{
45 Cifra propia obtenida por medio del levantamiento de datos realizado en el marco de la presente investigación, por medio del sistema SNIFA.

${ }^{46}$ Iván Poklepovic, “Análisis Crítico del Sistema de Incentivos al Cumplimiento Ambiental de la Ley 20.417, que crea la Superintendencia del Medio Ambiente," en Actas de las V Jornadas de Derecho Ambiental, Derecho Ambiental en tiempos de reformas (Santiago: 2011), 192.

${ }^{47}$ Kay Bergamini y Cristian Pérez, "Fiscalización y cumplimiento ambiental en Chile: principales avances, desafíos y tareas pendientes," EURE Vol.41, N 124 (2015): 273.

${ }^{48}$ Historia de la Ley 20.417, p.1492. “Aprobado un programa de cumplimiento por la Superintendencia, el procedimiento sancionatorio se suspenderá, a menos que cualquier tercero interesado o afectado por los efectos de la infracción se oponga fundadamente. De la oposición se dará traslado al infractor con cuyos descargos o sin ellos se resolverá la prosecución del procedimiento sancionatorio o el programa de cumplimiento. La resolución que resuelva la oposición o que establezca el programa de cumplimiento será apelable ante la Corte de Apelaciones respectiva".

${ }^{49}$ Luis Cordero, Lecciones de Derecho Administrativo (Santiago: LegalPublishing Chile, 2015), 365.
} 
observaciones a los programas de cumplimiento ${ }^{50}$ y la aportación de mayores antecedentes de los hechos contenidos en la formulación de $\operatorname{cargos}^{51}$-, ii) su rol fiscalizador del cumplimiento del instrumento -lo que se observa principalmente en los casos de reinicio de procedimiento sancionatorio en PPdC-, como también iii) su promoción del control jurisdiccional del actuar de la SMA en la aprobación de los PdC -mediante la interposición de reclamaciones ante los Tribunales Ambientales-.

En lo que se refiere a la fiscalización del PdC cabe destacar el procedimiento del Autódromo Codegua, donde fueron los mismos denunciantes que iniciaron el procedimiento sancionatorio -Junta de Vecinos $\mathrm{N}^{\circ} 199$ Reserva la Candelaria- los que, a un mes de haber aprobado el PdC, presentaron un escrito que informó la realización de una actividad en la cual se habrían sobrepasado los índices de presión sonora permitidos infracción que había dado lugar al PdC-. Luego, los mismos interesados realizaron una nueva presentación de denuncia dando aviso de una actividad en la que probablemente se incurriría en una nueva infracción. Posteriormente, la División de Fiscalización de la SMA constató, por medio de los informes aportados por la empresa, que siete de las ocho medidas comprometidas no fueron cumplidas adecuadamente. En este caso, el actuar activo del denunciante fue clave para determinar la existencia de una situación irregular, para luego decretar el incumplimiento del instrumento.

Como fue mencionado, la labor del denunciante se destaca también en la incitación del control jurisdiccional a la praxis administrativa referida a la aprobación de este instrumento. Así, la única impugnación de un PdC que ha tenido por resultado que un Tribunal Ambiental controle su contenido y finalmente lo modifique, es el caso R-36-2016 caratulado Víctor Alejandro Barría Oyarzo con Superintendencia del Medio Ambiente ante el Tercer Tribunal Ambiental, el cual fue iniciado por el denunciante de las infracciones. La impugnación recurrió contra la resolución que aprobó el PdC y suspendió el procedimiento sancionatorio, seguido por la SMA bajo expediente D-067-2015 en contra de la Empresa de Servicios Sanitarios Los Lagos S.A. (en adelante "ESSAL").

La reclamación, en lo medular, se funda en que el PdC aprobado por la SMA no cumplió con el criterio de aprobación positivo de integridad, lo anterior, motivado en que las labores de limpieza de un estero comprometidas por la empresa en su PdC no abarcaban todas las zonas afectadas por su actividad. El Tribunal Ambiental resuelve con un acogimiento parcial de la reclamación, manteniendo la resolución aprobatoria del PdC, pero ordenando a la SMA dejar sin efecto la parte que se refiere a la limpieza del estero,

\footnotetext{
${ }^{50} \mathrm{Al}$ respecto, el caso D-062-2015/Avícola Andina SpA, se irgue como un importante caso en el cual las observaciones del denunciante (Oliva Rutas del Sur) importan un antecedente a ponderar tanto por el infractor como por la SMA al momento determinar el contenido del PdC, tal como se indica en la Res. Ex. $\mathrm{N}^{\circ} 5$ del procedimiento

${ }^{51}$ Véase para tal efecto, Superintendencia del Medio Ambiente, D-063-2015/Pub Restaurante la Fraga.
} 
Galleguillos - Superintendencia del Medio Ambiente, Infractor y Denunciante en el Procedimiento de Programas de Cumplimiento Ambiental • Sección Doctrina

reemplazándola por un acto que se refiera de forma íntegra a la obligación de la empresa de limpieza ${ }^{52}$.

El caso descrito resulta de vital importancia por diversos motivos, entre ellos, por la consagración de la utilización de los principios de coherencia, realidad y precautorio en los procedimientos ante la SMA, pero especialmente, y para efectos de este estudio, destaca por ser un procedimiento motivado por un denunciante. Esta característica es relevante, ya que fueron los antecedentes otorgados por él, especialmente aquellos referidos a la existencia de impactos no subsanados por el programa, los que sirvieron de fundamento esencial para el pronunciamiento judicial, lo que permitió en definitiva que el instrumento se utilice de forma debida.

En suma, el actuar de los denunciantes ha sido de especial relevancia en la implementación de los PdC, a pesar de que la normativa aplicable no le otorgue un rol particular dentro de estos procedimientos, como tampoco establezca mecanismos de incentivo o facilitadores de su participación.

\section{Conclusiones}

En la segunda evaluación de desempeño ambiental de Chile realizada por la OCDE, el organismo internacional diagnóstica que nuestro marco institucional de físcalización sigue estando muy incompleto, asegurando que, a pesar de que la SMA dispone de una serie de mecanismos administrativos de fiscalización, su capacidad de acción es muy limitada $^{53}$. Esta apreciación externa de nuestro sistema da cuenta que, si bien la Ley $\mathrm{N}^{\circ}$ 20.417 significó un gran avance en lo que se refiere al fortalecimiento de las capacidades institucionales en materia ambiental -especialmente aquellas referidas a fiscalización-, es en su implementación y en la consolidación de los organismos creados con ella, donde podremos evidenciar con mayor nitidez los efectivos avances en esta materia.

El PdC, tal como fue enunciado al inicio de este trabajo, constituye un mecanismo de alta aplicación, y con ello una herramienta exitosa al momento de generar un real incentivo en los sujetos formulados de cargos. En este sentido, el PdC se ha ido consolidando y afianzando en sus criterios principalmente por medio de la práctica administrativa. En este escenario, se destacan como elementos que explican la exitosa implementación de los PdC: i) el rol activo de la SMA en lo que respecta a materializar su función de asistencia, en este aspecto se destaca la dictación de la GpPdC -en tanto este instrumento demuestra una clara intención de la SMA de uniformar criterios e incentivar la utilización del instrumento-; ii) un alto cumplimiento por parte de los sujetos regulados del $\mathrm{PdC}$, lo que ha legitimado la utilización de la herramienta como una efectiva forma de conseguir una rápida reconducción a la legalidad; iii) un apropiado diseño normativo en lo

\footnotetext{
${ }^{52}$ Esta decisión fue acordada con el voto en contra de Ministro Sr. Michael Hantke que estuvo por acoger la reclamación en todas sus partes declarando la ilegalidad de la resolución aprobatoria y ordenando la prosecución del procedimiento sancionatorio.

${ }^{53}$ OCDE, Evaluación de Desempeño Ambiental de Chile (CEPAL: 2016), 29.
} 
que refiere a los incentivos para la utilización de la herramienta por parte de los infractores; $\mathrm{y}$, finalmente, el que la SMA sea iv) un organismo fiscalizador altamente demandado y con bajos recursos, estimamos puede incentivar en el organismo la utilización de mecanismos más expeditos en la resolución de procedimientos sancionatorios. Estos factores dan cuenta que los inicios de implementación de este instrumento de colaboración han sido exitosos, en atención a los fines propuestos para el instrumento.

Por último, la investigación abordó el rol del denunciante y de la comunidad afectada por el proyecto o actividad, como sujetos no abordados de forma particular en el diseño normativo del PdC. A mi juicio, lo anterior constituye un error, dado que la práctica administrativa ha demostrado que estos actores tienen un rol vital en la mejor implementación de esta herramienta. Es por lo anterior, y teniendo en especial consideración la alta congestión del sistema de fiscalización ambiental, que el rol del denunciante y las comunidades afectadas debe ser redefinido y potenciado, otorgando incentivos y mecanismos facilitadores para su colaboración en la protección del medio ambiente.

\section{Bibliografía}

Bergamini, Kay y Cristian Pérez, "Fiscalización y cumplimiento ambiental en Chile: principales avances, desafíos y tareas pendientes" EURE Vol.41, N¹24 (2015).

Biblioteca del Congreso Nacional. Historia de la Ley $\mathrm{N}^{\circ} 20.417$ (2010): 13, http://www.bcn.cl/obtienearchivo?id=recursoslegales/10221.3/3929/6/HL20417.pdf.

Bermúdez, Jorge, "Fundamento y límites de la potestad sancionadora administrativa en materia ambiental", Revista de Derecho de la Pontificia Universidad Católica de Valparaíso, №.40 (2013). Bermúdez, Jorge, Fundamentos de Derecho Ambiental (Valparaíso: UV Ed., Segunda Edición, 2014).

Leal, Brigitte, "Planificación, colaboración y autocontrol: nuevos instrumentos normativos para la administración fiscalizadora," Revista de Derecho Público, Vol. 83 (Segundo Semestre, 2015).

Cordero, Luis, Lecciones de Derecho Administrativo (Santiago: LegalPublishing Chile, 2015).

Ezio Costa y Paula Fuentes, "La participación ciudadana en las declaraciones de impacto ambiental" Revista Justicia Ambiental Vol. III (2011): 83-106.

Dussaubat, Jean Paul, "Daño ambiental y el fracaso de la reparación voluntaria: diagnóstico y propuestas," Revista de Derecho Ambiental, Centro de Derecho Ambiental, №6 (2016).

Fiscalía Nacional Económica, Material de Promoción N³ referido a Programas de Cumplimiento en materia de Libre Competencia (Santiago: 2012).

Guzmán, Rodrigo, Derecho Ambiental Chileno. Principios, instituciones e instrumentos de gestión (Santiago: Planeta Sostenible, 2012).

Medina, Gonzalo, "Efectos del Compliance, Incentivos para un Buen Ciudadano Corporativo," La Semana Jurídica Thomson Reuters, Año II, Número 63 (2013).

Mirosevic, Camilo, "La participación ciudadana en el procedimiento de evaluación de impacto ambiental y las reformas introducidas por la Ley $\mathrm{N}^{\circ}$ 20.417," Revista de Derecho de la Pontificia Universidad Católica de Valparaíso Vol. XXXVI, N¹ (2011): 281- 323 
Galleguillos - Superintendencia del Medio Ambiente, Infractor y Denunciante en el Procedimiento de Programas de Cumplimiento Ambiental • Sección Doctrina

Ministerio del Medio Ambiente, "Acuerdo Regional Liderado por Chile espera dar mayor acceso a la ciudadanía a información y justicia ambiental" http://portal.mma.gob.cl/acuerdo-regionalliderado-por-chile-espera-dar-mayor-acceso-a-la-ciudadania-a-informacion-y-justicia-ambiental/.

OCDE, Evaluación de Desempeño Ambiental de Chile (CEPAL: 2016).

Ossandón, Jorge, Incentivos al Cumplimiento Ambiental (Santiago: LibroMar, 2015), 475.

Plumer, Marie Claude, "Los Tribunales Ambientales: se completa la reforma a la institucionalidad ambiental" Anuario de Derecho Público, Universidad Diego Portales (2013).

Poklepovic, Iván, "Análisis Crítico del Sistema de Incentivos al Cumplimiento Ambiental de la Ley 20.417, que crea la Superintendencia del Medio Ambiente." En Actas de las V Jornadas de Derecho Ambiental, Derecho Ambiental en tiempos de reformas (2010).

Superintendencia del Medio Ambiente, "Guía para la Presentación de Programas de Cumplimiento Ambiental por Infracciones a Instrumentos de Carácter Ambiental," julio 2016, http://www.sma.gob.cl/index.php/documentos/documentos-de-interes/documentos/guias-sma.

Superintendencia del Medio Ambiente, "Guía para la presentación de un Programa de Cumplimiento Infracciones a la norma de emisión de ruidos, Infractores de Menor Tamaño" http://www.sma.gob.cl/index.php/documentos/documentos-de-interes/documentos/guias-sma.

\section{Leyes y normas}

Ley $\mathrm{N}^{\circ}$ 19.300, sobre Bases Generales del Medio Ambiente.

Ley N²0.393 sobre Responsabilidad Penal de las Personas Jurídicas por delitos de cohecho de funcionario público nacional o extranjero, lavado de activos y financiamiento del terrorismo.

Decreto Supremo No 30, 2013, Ministerio del Medio Ambiente, que aprueba el Reglamento sobre Programas de Cumplimiento, Autodenuncia y Planes de Reparación.

\section{Jurisprudencia judicial}

Corte Suprema, Rol $\mathrm{N}^{\circ}$ 1.119-2015. Fernando Dougnac y otros con Ministerio del Medio Ambiente. 30 de septiembre de 2015.

Segundo Tribunal Ambiental, Rol No R-68-2015. Sociedad Vinícola Miguel Torres S.A. en contra de la Superintendencia del Medio Ambiente (Res. Ex.N ${ }^{\circ}$ 12/Rol N ${ }^{\circ}$ D-26-2014, de 20 de abril de 2015), 22 de julio 2016.

Recibido: 13-12-2016

Aceptado: 24-6-2017 